\section{Fulfilling a lifelong dream}

\author{
Bryant S. Blank, DVM, MS, Clinical Veterinarian, Cornell University Center for \\ Animal Resources and Education, Cornell University, Ithaca, NY
}

\begin{abstract}
Dr. Blank tells Lab Animal about how he got involved in veterinary sciences and lab animal research.
\end{abstract}

\section{You earned a B.S. in Biology with a minor in Animal Sciences \& Industry. What first led you into animal science, and how did that guide your path towards becoming a clinical veterinarian?}

I grew up in a small city in the Midwest. I was not exposed to a lot of agriculture growing up, despite my proximity to it, so my lifelong goal of becoming a veterinarian involved a specific idea of the pets I would be caring for. Some early veterinarian mentors emphasized the importance of understanding all species of animals, not just companion animals, and guided me to my initial choices of an undergraduate field of study. While I went on to major in Biology (I really missed some of the basic biological sciences), I know that the exposure to both agricultural animals and the people who dedicate their lives to them broadened my horizons and helped me immensely in veterinarian school. It also continues to influence me in my current position where I am often involved in agricultural research studies.

\section{Please describe a typical workday for} you, highlighting your favorite aspects. I always laugh when I get this question because no day is typical and that is a good thing. My duties vary greatly and involve procedural and surgical support for research projects, reviewing IACUC protocols, performing facility rounds and clinical duties, and mentoring residents and veterinary students. For me the most rewarding aspect is when I can help a researcher with an issue or concern, ultimately making their life easier, the environment of open communication.

You have an impressive publication record. How did you get involved in research?

Impressive may be a stretch compared to many people I have worked with, but I do try to take every opportunity I can to collaborate on a wide variety of projects. My choice of a career in this field was initially about the animals and the people, but I have developed a real appreciation and enjoyment of being a part of some of the amazing research going on around me. Through opportunities in my Master's program and collaborative projects, I have had some amazing research mentors - from PIs and veterinarians to laboratory technicians. I have hopefully provided as much benefit to the projects I have worked on as I have gained from them.

\section{What advice would you give students} and younger colleagues considering the same career path?

Take every opportunity you can to see as many new things, new places and new people in this field as possible. A personal concept of the ideal position changes drastically over time for most people. The more you experience the better you will be as a residency candidate and ultimately lab animal veterinarian (and hopefully a happier and more fulfilled individual as well). Also, animal happier and encouraging an

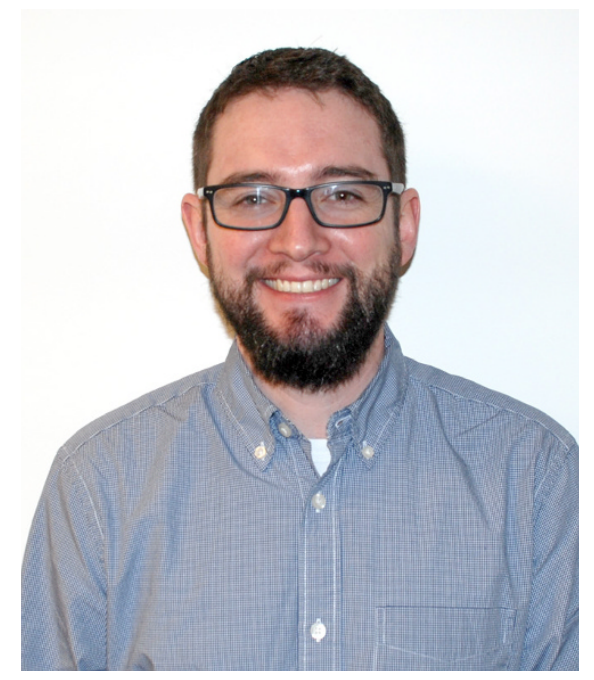

reach out to those of us already in the field. Most of us love to mentor and share our experiences. In this small world of lab animal science, you never know when those connections will change your life.

\section{Is there anything else you would like readers of Lab Animal readers to know about?}

The main piece of wisdom that I feel I can share with people in this field is that being humbled on daily basis is a good thing. I am consistently reminded of how little I know about a wide variety of things. The beauty of lab animal medicine is that there are so many dedicated people across the country and beyond who are willing to share and teach to improve the field and the care of the animals with which we work. In my current position I regularly go to colleagues for guidance, including other veterinarians, technicians, managers and husbandry staff. We all have strengths that make us assets. The willingness of so many to share those strengths, while continuing to improve on our weaknesses, is what makes the people in this field amazing and truly epitomizes the definition of 'lifelong learners'. 